\title{
Sources, Injection and Acceleration of Heliospheric Ion Populations
}

\author{
George Gloeckler ${ }^{1,2}$, Lennard A. Fisk ${ }^{2}$, Thomas H. Zurbuchen ${ }^{2}$ and \\ Nathan A. Schwadron ${ }^{2}$ \\ ${ }^{I}$ Department of Physics and IPST, University of Maryland, College Park, Maryland 20742 \\ ${ }^{2}$ Department of Atmospheric, Oceanic and Space Sciences, University of Michigan, \\ Ann Arbor, Michigan 48109-2143
}

\begin{abstract}
A variety of heliospheric ion populations -- from Anomalous Cosmic Rays (ACRs) to particles accelerated in Corotating Interaction Regions (CIRs) -- have been observed and studied for several decades. It had been commonly assumed that the solar wind was the source for all of these populations, except for the ACRs, and that shock acceleration produced the energetic particles observed, including the ACRs. For the ACRs the source that had been proposed a long time ago was the interstellar gas that penetrates deep into the heliosphere. Recent measurements of the composition and spectra of suprathermal ions, primarily from Ulysses, ACE and Wind, indicate that pickup ions are likely to be an important source not only of the ACRs but for other heliospheric ion populations as well. In particular, the newly discovered "Inner Source" pickup ions may be a significant source for particles accelerated in the inner heliosphere and may also be the seed material for ACR C, Mg, Si and Fe. Furthermore, the omnipresent suprathermal tails seem to tell us that shock acceleration may not be the primary mechanism energizing particles to $\sim 0.1$ $\mathrm{MeV}$ in the heliosphere. Explaining the origin of these persistent high velocity tails remains one of our challenges.
\end{abstract}

\section{INTRODUCTION}

Observational tools available to us today make it now possible to begin detailed exploration of acceleration mechanisms that produce a variety of heliospheric particle populations that were discovered during the last four decades. The key questions addressed, in addition to the acceleration processes, concern sources of accelerated particles and injection mechanisms. There is a clear advantage to observe these processes where they take place. What we learn in our heliosphere will have important implications to processes in other astrophysical settings for which detailed local observations are not possible.

Instruments on ACE and Ulysses provide us with the most comprehensive elemental and charge state composition measurements to date. The energy range covered by these instruments extends from typical solar wind energies $(\sim 1 \mathrm{keV} / \mathrm{amu})$ to tens of MeV/amu. Thus velocity distributions of both the source material and the accelerated particles may be simultaneously obtained. In this report we confine ourselves to observations made with the SWICS instruments $(1,2)$ on ACE and Ulysses, that measure the source populations from 1 to $5.4 \mathrm{AU}$, in the ecliptic and high latitudes, and cover the important velocity range where injection and the initial acceleration (pre-acceleration) occurs. SWICS measures elemental abundances, charge-state composition and speed distribution functions of ions from 0.6 to $60 \mathrm{keV} / \mathrm{e}$ on Ulysses and from 0.6 to $\sim 100 \mathrm{keV} / \mathrm{e}$ on ACE.

\section{OBSERVATIONS OF SOURCES}

In order to determine which populations are the source of energetic particles produced in an acceleration process it is necessary first to establish the characteristics of these populations. Each source population has special characteristics which separate one from the other.

\section{Solar Wind during Quiet Times}

Shown in Figure 1 are the speed distribution functions (in the spacecraft frame) for hydrogen and helium averaged over a 65 day time period observed at $1 \mathrm{AU}$ with SWICS on ACE. Time intervals around shocks and when magnetic clouds, counter-streaming electron events and magnetic holes were observed have been specifically excluded from these averaged distributions. It is remarkable that all three spectra, including $\mathrm{He}^{+}$ pickup ions, have pronounced high-speed tails that approach a power law and persist even during time periods when no shocks and other disturbances ane observed locally. 


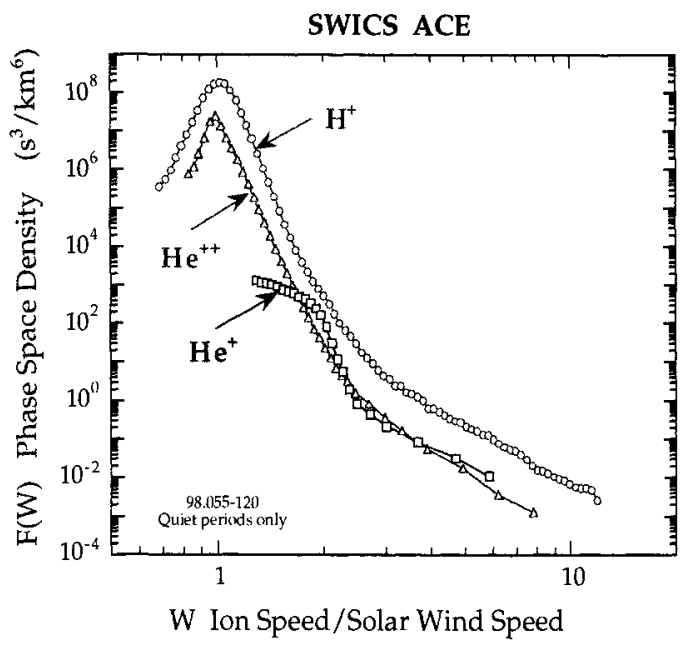

FIGURE 1. Speed distributions of $\mathrm{H}^{+}, \mathrm{He}^{++}$and $\mathrm{He}^{+}$ observed in the quiet in-ecliptic solar wind at 1 AU. These spectra were measured with the SWICS instrument on ACE and averaged over the 65 day observation period (24 February to 30 April, 1998). Time periods around shocks or other disturbances were excluded from these averaged spectra (times of disturbances in the solar wind can be found at http://www.bartol.udel.edu/ chuck/ace/ACElists/obs_list.html).

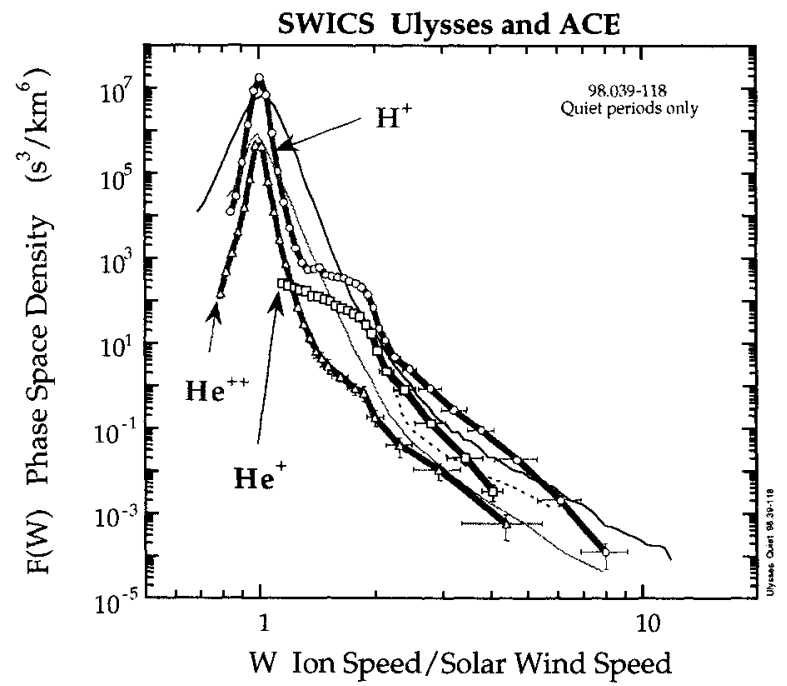

FIGURE 2. Speed distributions of $\mathrm{H}^{+}, \mathrm{He}^{++}$and $\mathrm{He}^{+}$ (bold curves) observed in the quiet low-latitude solar wind at 5.4 AU with the SWICS instrument on Ulysses for approximately the same time period as in Figure 1. Ulysses and $A C E$ were nearly radially aligned during this time. Disturbed time periods (e.g. around shocks, Forsyth and Gosling, private communication) are excluded from these averaged distribution functions. Shown for comparison from Figure 1, as the two thin curves, are the speed distribution functions of $\mathrm{H}^{+}$and $\mathrm{He}^{++}$, reduced by 29.2 to account for the $R^{-2}$ decrease in the solar wind density with heliocentric distance $R$, and of $\mathrm{He}^{+}$, as the dashed curve, reduced by 8.5 in order to match the Ulysses spectrum below $W \approx 2$.
Figure 2 shows the velocity distributions of $\mathrm{H}^{+}$, $\mathrm{He}^{++}$and $\mathrm{He}^{+}$observed at $5.4 \mathrm{AU}$ with SWICS on Ulysses during a time interval comparable to that in Figure 1. Ulysses, nearly radially aligned with $\mathrm{ACE}$ and the Sun during this time period, was sampling more or less the same solar wind as ACE. Again, time intervals around shocks and other disturbances were excluded from these averaged distributions. At $5.4 \mathrm{AU}$ one observes, not only the solar wind ions and pickup $\mathrm{He}^{+}$seen at $1 \mathrm{AU}$, but, in addition, interstellar pickup $\mathrm{H}^{+}$and $\mathrm{He}^{++}$at $W$ (ion speed divided by the solar wind speed) above $\sim 1.5$. Again, each of the velocity distributions at $5.4 \mathrm{AU}$ has a well-developed tail. Shown for comparison are also the scaled distribution functions from Figure 1 (see caption of Figure 2) of $\mathrm{H}^{+}$and $\mathrm{He}^{++}$. Comparing the $1 \mathrm{AU}$ spectra to the corresponding $5 \mathrm{AU}$ distributions, we find that at $\sim 5 \mathrm{AU}$ the densities in the tail region $(W>\sim 2)$ relative to the bulk of the distribution (around $W \approx 1$ ) are about the same as at $1 \mathrm{AU}$, despite significant cooling clearly evident in the solar wind distributions. This implies that the tails are continuously regenerated in the out-flowing solar wind to overcome cooling of the distributions in the expanding solar wind. It should also be noted that at $5.4 \mathrm{AU}$, unlike at $1 \mathrm{AU}$, the density of $\mathrm{He}^{+}$for $W$ above $\sim 1.25$ is much larger than that of $\mathrm{He}^{++}$.

\section{Interstellar Pickup Ions}

Interstellar pickup ions are created by ionization of slow moving interstellar atoms inside the heliosphere $(3,4)$. The pickup process produces suprathermal velocity distributions with a characteristic cut-off at twice the solar wind speed, $W \approx 2$. At 1 AU only inter-

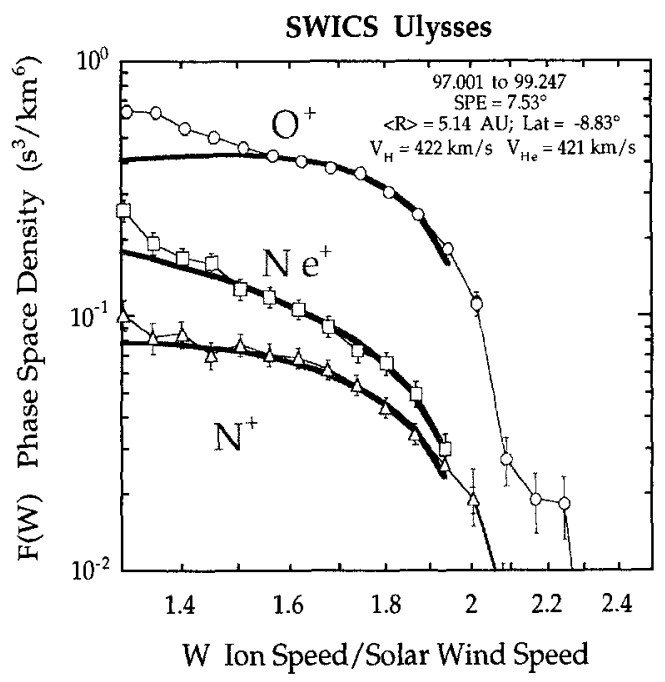

FIGURE 3. Time-averaged speed distributions of $\mathrm{N}^{+}, \mathrm{O}^{+}$ and $\mathrm{Ne}^{+}$observed at $5.4 \mathrm{AU}$ with SWICS on Ulysses during the 2.68 year time period starting 1 Jan., 1997. The bold curves are model calculations of the predicted spectra. 
stellar pickup $\mathrm{He}^{+}$(see Figure 1) is readily observed because atomic $\mathrm{He}$, being difficult to ionize, penetrates closer to the Sun than $\sim 0.5 \mathrm{AU}(3,4)$. Beyond several $\mathrm{AU}$, however, other interstellar pickup ion species begin to appear. At 5.4 $\mathrm{AU}$ interstellar pickup $\mathrm{H}^{+}$and $\mathrm{He}^{++}$ are visible above the suprathermal extension of the respective solar wind distributions (see also refs. 5 and 6). The velocity distributions of the heavy interstellar pickup ions, $\mathrm{N}^{+}, \mathrm{O}^{+}$and $\mathrm{Ne}^{+}$, are presented in Figure 3 . The spectra of these ions all have the characteristic cutoff at $W \approx 2$. It is fairly straightforward to model the velocity distributions and predict the spatial profiles of the density of interstellar pickup ions $(3,6,7)$. The shape of the velocity spectrum of a particular pickup ion species depends on the loss rate of the corresponding interstellar atoms, and the pickup ion density is the product of the production rate and the interstellar atomic density at the heliospheric termination $\operatorname{shock}(5,6)$. For distribution functions that are averaged over time periods of about one year or more the production and loss rates become approximately equal. In Figure 3 the fits to the data (bold curves) in the interval $\sim 1.5<W<\sim 2$ were used to determine ionization loss rates. The densities of the corresponding interstellar atomic species at the termination shock are then found using the production rates that are assumed to be equal to the corresponding loss rates(6).

\section{Pickup Ions from the Inner Source}

Creation of interstellar pickup ions requires the presence of interstellar atoms deep in the heliosphere. Elements already ionized in the interstellar medium (for example $\mathrm{C}^{+}$) flow around the heliopause and do not enter the heliosphere. Interstellar pickup ions of these elements are therefore not expected and have not been found. However, Geiss et al.(8) found amounts of pickup $\mathrm{C}^{+}$comparable to $\mathrm{O}^{+}$in the mass spectrum of singly-charged heavy ions with speeds between $\sim 0.8$ and 1.2 times the solar wind speed. This indicated another source for pickup ions which they called the "inner source" $(8,9)$.

Clear evidence for the existence of the inner source is provided in Figure 4 which shows the distribution functions of $\mathrm{O}^{+}, \mathrm{C}^{+}$and $\mathrm{Ne}^{+}$observed with SWICS on Ulysses at low and middle latitudes at an average heliocentric distance of $\sim 1.5 \mathrm{AU}(10)$. This close to the Sun, the density of interstellar pickup $\mathrm{O}^{+}$and $\mathrm{Ne}^{+}$is small because of removal of interstellar oxygen and neon atoms by ionization. Yet all three spectra show orders of magnitude higher densities at $W$ around 1 then near the $W \approx 2$ cut-off. While $\mathrm{C}^{+}$has no detectable interstellar component, as is expected, its density at $W$ between $\sim 0.8$ and $\sim 1.2$ is about the same as that of $\mathrm{O}^{+}$.

The distribution functions of inner source pickup ions have certain characteristics: (1) they peak at or below the solar wind peak at $W=1$, and (2) they are relatively cold, in that their effective thermal speed would be small compared to the solar wind speed, in clear contrast with the usual interstellar pickup ion distributions. The interstellar pickup ion distribution is quite broad and extends with nearly equal phase space density up to $W \approx 2$, beyond which the density drops $(3,4)$. Interstellar pickup ions gyrate about the magnetic field in the solar wind immediately following their ionization(3). Although initially they will propagate inward in the frame of the solar wind, upon scattering they can reverse direction, in which case they can acquire speeds (in the spacecraft reference frame) up to the sum of twice the solar wind speed and the initial speed of the slow moving (compared to the solar wind) neutrals: that is, $W \approx 2$.

The same process should occur for the inner source pickup ions. However, they are most likely produced from neutrals emitted from heliospheric grains $(10,11)$. Because neon is clearly present in the inner source pickup ions it is believed that the emitted neutrals

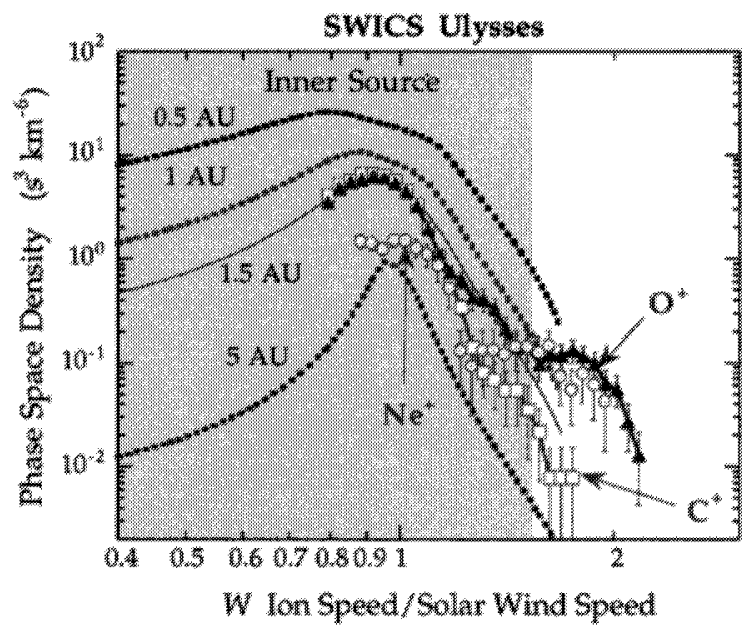

FIGURE 4. Time-averaged speed distributions of $\mathrm{C}^{+}, \mathrm{O}^{+}$ and $\mathrm{Ne}^{+}$observed from November 21, 1994 to May 30, 1995 at low and middle latitudes $\left(<60^{\circ}\right)$ and $1.5 \mathrm{AU}$ with SWICS during the fast latitude scan of Ulysses. The distributions are plotted versus $W$, the speed of the ions relative to the solar wind speed, in a stationary reference frame relative to the spacecraft. The four curves are model calculations for $\mathrm{O}^{+}$and show the effects of strong cooling as evidenced by the narrowing of the peaks at $W \sim 0.9$ with increasing distance.

include 're-cycled' solar wind material. Solar wind ions are constantly absorbed by the grains and then re-emitted as slow moving atoms and molecules. Schwadron et al.(11) have shown that the absorption, re-emission, and ionization processes that produce the observed inner source pickup ion distributions occur near the Sun, and hence the inner source pickup ions suffer substantial cooling. The distribution functions in Figure 4 are 
clearly weighted toward speeds $W<1$. The particles thus suffer relatively little scattering and retain their inward motion in the frame of the solar wind(7). The expansion of the solar wind, however, tends to reduce the particle speeds perpendicular to the magnetic field and substantially cool the distributions(11).

Modeling of the expected velocity distributions of the inner source pickup ions and matching them to the spectra observed at some fixed distance from the Sun allows us to estimate the radial profiles of the density of the neutral particles that produce a given inner source pickup ion species(11). Then we can predict the pickup ion distribution functions at other distances and the radial profile of pickup ion density. The model curves shown in Figure 4 represent the predicted velocity distributions of oxygen at the indicated radial distances

Other pickup ion populations that could potentially become a source of accelerated energetic particles include planetary and cometary pickup ions. These are, however, very local sources, with their extended plasma tails confined to within several degrees of a line connecting the Sun and the planet or comet(12). The composition of these pickup ion populations is highly variable. For example, there is virtually no $\mathrm{C}^{+}$in the Earth's tail, while lowly charged sulfur ions are abundant in Jupiter's plasma tail. The composition of pickup ions in distant cometary tails seems to be the same for the few comets that have so far been studied with in situ mass spectrometers(12). For cometary pickup ions the abundance of nitrogen is low, and neon is extremely rare.

\section{Comparison of Abundances of Source Populations}

Except for the inner source, Figure 5 shows the total abundance of each source relative to its oxygen abundance. For the inner source, the abundance is relative to neon and divided by 10 for ease of comparison. Relative abundances of the three source populations $(6,10)$ and of cometary tail pickup ions(12) are compared in Figure 5 to the sample-averaged composition of energetic $(\sim 2$ to $20 \mathrm{MeV} / \mathrm{amu})$ particles accelerated in Corotating Interaction Regions, CIRs(13,14). From comparison of the composition alone it is clear that a cometary source can be ruled out as a major contributor to the CIR accelerated energetic particles. This is not surprising because cometary sources are localized and hence do not add much to the source populations of CIR particles. On the other hand, some combination of roughly equal amounts from the three other sources (solar wind, interstellar and inner source pickup ions) could reproduce the CIR composition within a factor of two. We would therefore argue that using the similarity in compositions as the only criterion for deciding on the source material for accelerated particles is insufficient.

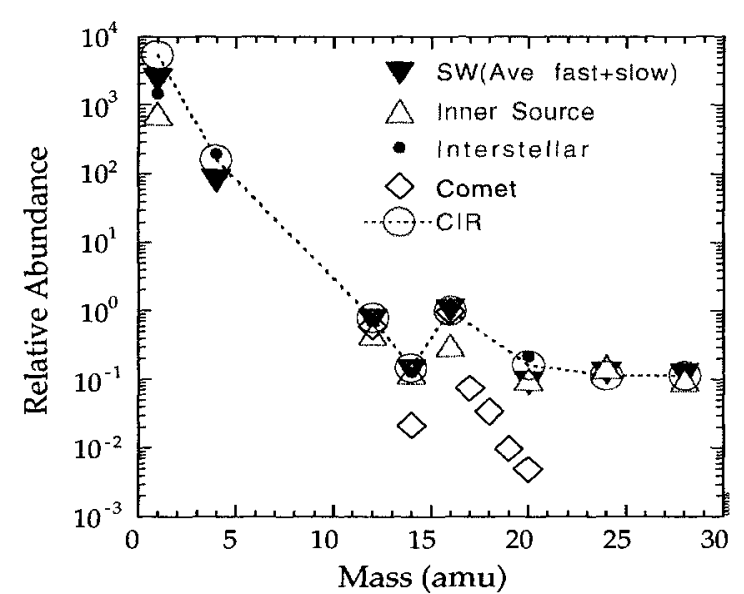

FIGURE 5. Relative abundances from four potential sources of energetic particles compared to the average composition of CIR accelerated particles.

\section{Velocity Distributions in the Turbulent Solar Wind}

Speed distributions of $\mathrm{H}^{+}, \mathrm{He}^{+}$and $\mathrm{He}^{++}$were measured(15) with SWICS/Ulysses downstream of the forward (FS) and reverse (RS) shocks in the CIR of late December 1992. Here we summarize several of the remarkable features apparent from the speed spectra shown in Figure 7 of reference 15. First, it was found that more $\mathrm{He}^{+}$than $\mathrm{He}^{++}$is accelerated even though solar wind alpha particles are at least a factor of $10^{3}$ more abundant than pickup $\mathrm{He}^{+}$. Second, the spectral shapes in the high-speed ( $W>2.4$ ) tails behind the reverse shock were identical (within experimental uncertainties) to those behind the forward shock, even though the two shocks were different. The FS was weaker than the RS, and one was quasi-perpendicular while the other quasiparallel(16). It was also evident from the speed distributions that the solar wind behind the reverse shock was heated more than the wind downstream of the weaker forward shock.

Downstream of the CIR reverse shock the shapes of the speed distributions above $W \sim 2.4$ were found to be identical (within experimental uncertainties) for all three species. This, combined with the fact that the FS and RS spectra also had the same shapes, implies that the injecting/pre-acceleration mechanism depends primarily on ion speed. In addition, the spectral shapes were found not to be simple power laws as would be predicted by standard shock acceleration models, providing further evidence that the high-velocity tail distributions that had been observed were not produced by a simple shock acceleration mechanism(17).

Figure 6 shows the speed distribution of $\mathrm{H}^{+}, \mathrm{He}^{+}$ and $\mathrm{He}^{++}$measured with SWICS/ACE downstream of the large forward shock which occurred at $\sim 0215$ on May 


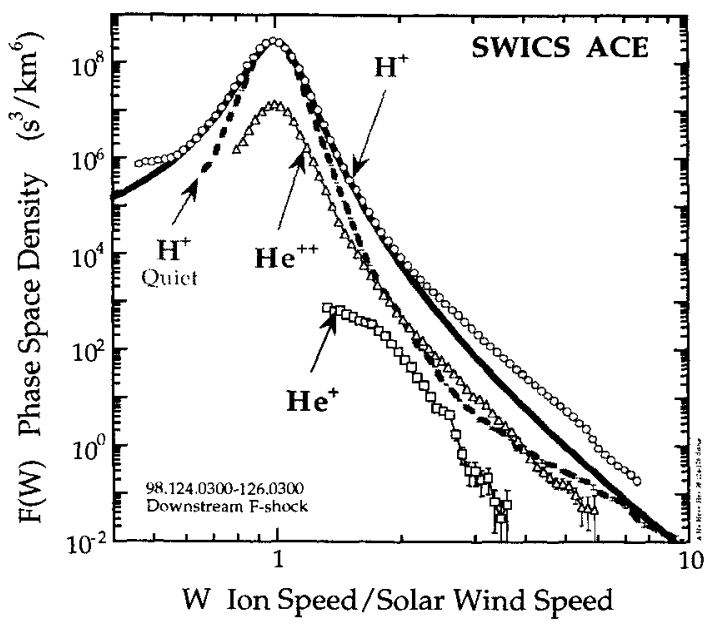

FIGURE 6. Speed distributions of $\mathrm{H}^{+}, \mathrm{He}^{++}$and $\mathrm{He}^{+}$observed during a two-day period (4 May 0300 to 6 May 0300,1998 ) behind the large forward shock of 4 May, 1998. The quiet-time proton spectrum from Figure 1 is shown for comparison (dashed curve). The density of $\mathrm{He}^{+}$ is below that of $\mathrm{He}^{++}$for all $W$ and its tail is steeper than that $\mathrm{He}^{++}$.

4, 1998. The kappa function(6) shown as the solid curve, $(\kappa=3.2)$ is an excellent fit to the observed $\mathrm{H}^{+}$ spectrum for $\sim 0.6<W<\sim 1.5$. Outside this range, the proton density is above the kappa fit. The solar wind behind this strong shock is both hotter and has well developed tails compared to the solar wind during quiet times (see Figure 1 and dashed curve of Figure 6). The density of interstellar $\mathrm{He}^{+}$is well below that of the solar wind for all $W$. This is in contrast to the quiet-wind cases both at $1 \mathrm{AU}$, where the $\mathrm{He}^{+}$and $\mathrm{He}^{++}$densities are comparable in the tail regions, and at $5.4 \mathrm{AU}$, where the $\mathrm{He}^{+}$density above $W>\sim 1.25$ is larger than the $\mathrm{He}^{++}$density. The tail of $\mathrm{He}^{+}$downstream of the shock is steeper than the solar wind $\mathrm{H}^{+}$and $\mathrm{He}^{++}$tails.

\section{Injection Efficiency}

Knowledge of the injection efficiencies and of the distribution functions (and not just the composition) of the source populations is required in order to determine the origin of accelerated particles. Injection efficiencies are not easily defined and difficult to measure or compute. Here we use a simple definition of the injection efficiency which will allow us to use measurements of the speed distribution of ions in the turbulent solar wind (e.g. downstream of a shock) to estimate injection efficiencies. Consider an ion of a certain species moving at some given speed, $w_{i}$, where $w_{i}$ is the ion speed in the solar wind frame divided by the solar wind bulk speed. We define this ion to be injected for further acceleration (e.g. by a shock) if its speed is increased to a value above some given threshold speed, $w_{t}$. The injection efficiency, $\mathrm{I}\left(w_{i}\right)$, is thus defined to be the

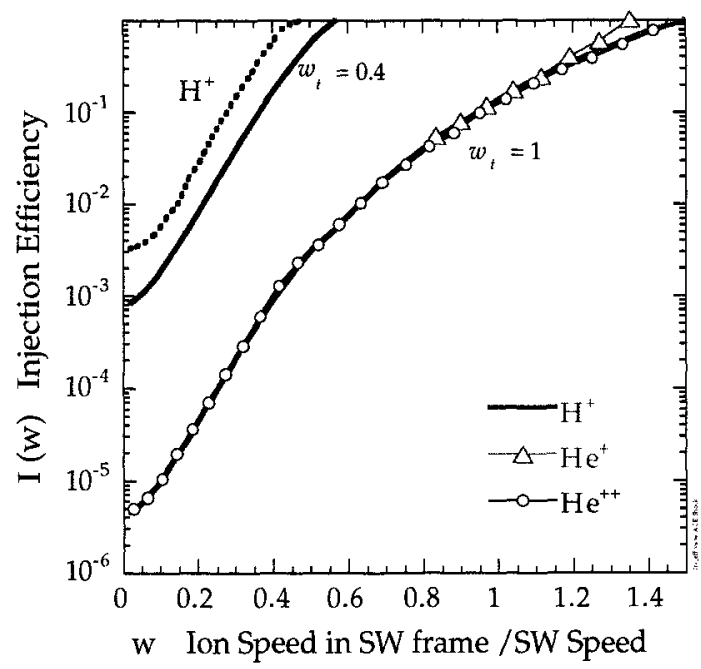

FIGURE 7. Injection efficiency vs. normalized ion speed in the solar wind frame for $\mathrm{H}^{+}, \mathrm{He}^{++}$and $\mathrm{He}^{+}$derived from speed distributions given in Figure 6 in the downstream region of the 4 May, 1998 shock. See text for description of the method used to compute the efficiencies.

integrated phase space density of all ions of that species above the threshold speed $w_{t}$, divided by the product of the phase space density of ions at $w_{i}$, times the number of $w_{i}$ steps below $w_{t}$. With this definition we assume that the initial distributions have much lower density above $w_{t}$ than the final distributions. This seems to be the case if the initial distributions are similar to the quiet-time spectra (e.g. compare in Figure 6 the quiettime $\mathrm{H}^{+}$spectrum to the corresponding $\mathrm{H}^{+}$distribution in the downstream region).

Figure 7 shows the injection efficiencies of $\mathrm{H}^{+}, \mathrm{He}^{+}$ and $\mathrm{He}^{++}$for $w_{t}=1.0$, and also $\mathrm{H}^{+}$for $w_{t}=0.4$, computed as defined above using the speed distribution functions shown in Figure 6 . The turbulent solar wind behind the shock was chosen because ions from distributions in this region are most likely to be injected for further acceleration at the shock. For injection threshold speeds of $w_{t}=0.4$ we were able to estimate $\mathrm{I}\left(w_{i}\right)$ for protons moving inward in the solar wind frame ( $W<1$, toward the Sun, dotted curve) as well as for those moving outward ( $W>1$, solid curve). It appears that the inward-moving protons are injected more efficiently than those moving outward. For a 0.4 injection threshold speed the injection efficiency for thermal solar wind protons (those with $w<\sim 0.1$, a typical value of the thermal speed divided by the bulk speed) is a fraction of one percent.

For $w_{t}=1$ and ions moving outward, $\mathrm{I}\left(w_{i}\right)$ was computed for $\mathrm{H}^{+}$(solid curve), $\mathrm{He}^{+}$(solid triangles) and $\mathrm{He}^{++}$(open circles). The three efficiency curves are nearly identical, implying a purely speed-dependent injection process with no dependence on the mass or mass/charge of the ion. With the injection threshold speed equal to the bulk solar wind speed $(-630 \mathrm{~km} / \mathrm{s}$ in 
this case) the injection efficiency of thermal $(w<\sim 0.1)$ solar wind ions is about $10^{-5}$. This very low efficiency for injecting thermal solar wind ions is consistent with our observation (shown in Figure 6) that the density of $\mathrm{He}^{+}$in the tail $(W>\sim 2)$ is only about an order of magnitude lower than the tail density of $\mathrm{He}^{++}$while at $W=\sim 1$ the density of $\mathrm{He}^{++}$is about $10^{4}$ higher than that of $\mathrm{He}^{+}$. Because all efficiencies increase with increasing $w$, suprathermal ions, such as interstellar pickup ions, are expected to be preferentially injected.

\section{Radial Variation of the Oxygen Density from Different Source Populations}

The amount of material a particular source contributes to accelerated particle populations will depend on how far from the Sun the acceleration takes place. Because the density of interstellar pickup ions peaks at large heliocentric distances $(\sim 5 \mathrm{AU}$ or more, except for $\mathrm{He})$, interstellar pickup ions will be an important source of particles accelerated in the outer heliosphere. Conversely, because the density of the solar wind and of inner source pickup ions is highest close to the Sun these ions will contribute most of the material for particles accelerated in the inner heliosphere. The radial dependence of the density of oxygen from the three sources is shown in Figure 8. The density profile of inner source $\mathrm{O}^{+}$is derived from best fits of the model distribution function to the observed velocity distribu-

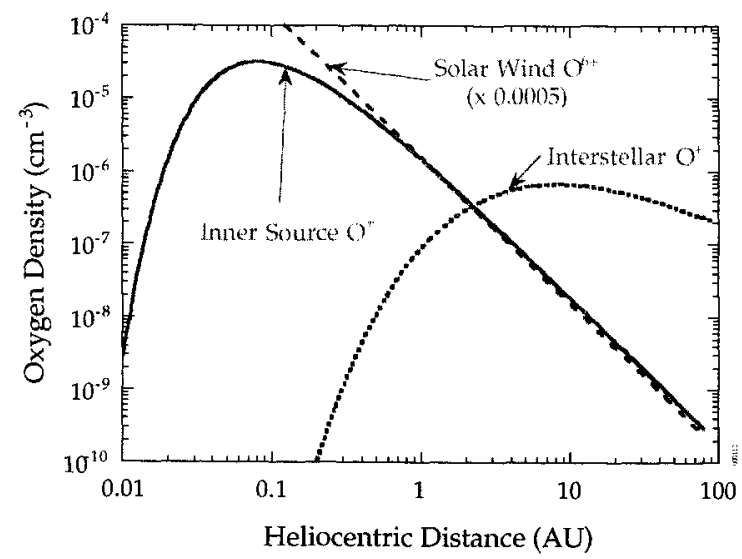

FIGURE 8. Predicted variation with heliocentric distance of the densities of inner source $\mathrm{O}^{+}$, interstellar pickup $\mathrm{O}^{+}$ and solar wind $\mathrm{O}^{6+}$. For better comparison we reduced the solar wind oxygen density by a factor of 2000 .

tion of $\mathrm{O}^{+}$(solid curve in Figure 4). The solar wind $\mathrm{O}^{6+}$ density (dashed line) has been reduced by a factor of 2000 to aid in comparison between the three distributions. The interstellar $\mathrm{O}^{+}$density was computed using ionization loss rate and atomic oxygen density obtained from best fits to the velocity distribution of $\mathrm{O}^{+}$ shown in Figure 3.
The importance of interstellar pickup ions beyond several AU is illustrated in Figure 8. There the density of interstellar $\mathrm{O}^{+}$exceeds that of the inner source and the solar wind, provided that efficiency of injecting thermal solar wind $\mathrm{O}^{6+}$ is less than $5 \cdot 10^{-4}$. Such injection efficiencies for thermal ions are not unreasonable, and, as can be seen from Figure 7, would indicate an injection threshold speed of $w_{t} \approx 0.5$. These calculated density profiles in Figure 8 also indicate the likelihood that the source for Anomalous Cosmic Ray (ACR) carbon (as well as $\mathrm{Si}$ and other elements that are already ionized in the Local Interstellar Cloud) is the inner source. In the inner source the abundance of $\mathrm{C}^{+}$is comparable to $\mathrm{O}^{+}$ (see Figures 4 and 5). The $\mathrm{O} / \mathrm{C}$ ratio measured in the ACR is about 160(18). From Figure 8 we find that the density ratio of interstellar $\mathrm{O}^{+}$to inner source $\mathrm{C}^{+}$ (or $\mathrm{O}^{+}$) should be 160 at about $30 \mathrm{AU}$.

\section{Comparison of Velocity Distributions of Source Populations}

In deciding on the source of a given energetic particle population it is not sufficient to compare the relative abundance of the accelerated ion population to that of one of the source populations. When two sources have a similar composition one must examine their velocity distributions at a given location in order to decide which is preferentially injected. In Figure 9 we compare the predicted distribution functions of inner source $\mathrm{O}^{+}$(from Figure 4) and interstellar $\mathrm{O}^{+}$at $1 \mathrm{AU}$ with the velocity distribution of solar wind $\mathrm{O}^{6+}$ measured with $\mathrm{ACE}$ at 1 AU. The phase space density of solar wind oxygen exceeds that of the other two sources for $W>\sim 0.6$. For $W<\sim 0.6$ the inner source $\mathrm{O}^{+}$density is larger than that

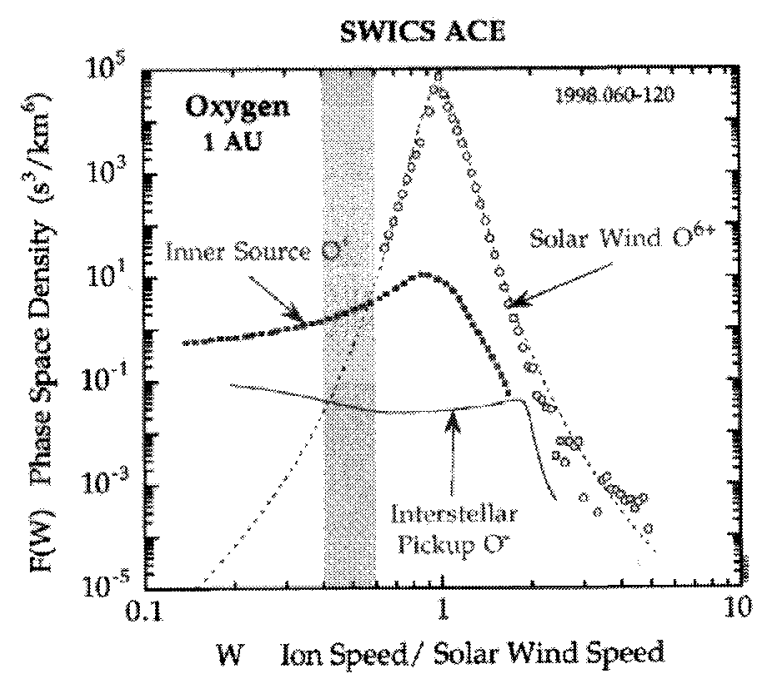

FIGURE 9. Comparison of the predicted velocity distributions, at $1 \mathrm{AU}$, of inner source $\mathrm{O}^{+}$and the interstellar $\mathrm{O}^{+}$with the solar wind $\mathrm{O}^{6+}$ spectrum measured with SWICS on ACE during the same time period as in Figure 1. 
of the other two sources. For particles accelerated at $1 \mathrm{AU}$, interstellar $\mathrm{O}^{+}$is not a significant source since its density is below the other two sources for all speeds. The relative contributions of the other two sources to energetic particles accelerated at $1 \mathrm{AU}$ will depend critically on the injection efficiency, which depends on ion speed $W$ (see Figure 7). Thus, assuming for simplicity that injection efficiency is 0 for $\sim 0.6<W<\sim 1.5$ and 1 for all other $W$, then inner source $\mathrm{O}^{+}$will be the principal source of accelerated particles. However, if the injection efficiency is zero only inside the smaller interval $\sim 0.75<W<\sim 1.3$ then the solar wind will be the dominant source.

The situation is entirely different at other locations in the heliosphere. In Figure 10 we show what happens at $5 \mathrm{AU}$. Again we compare the predicted spectra of inner

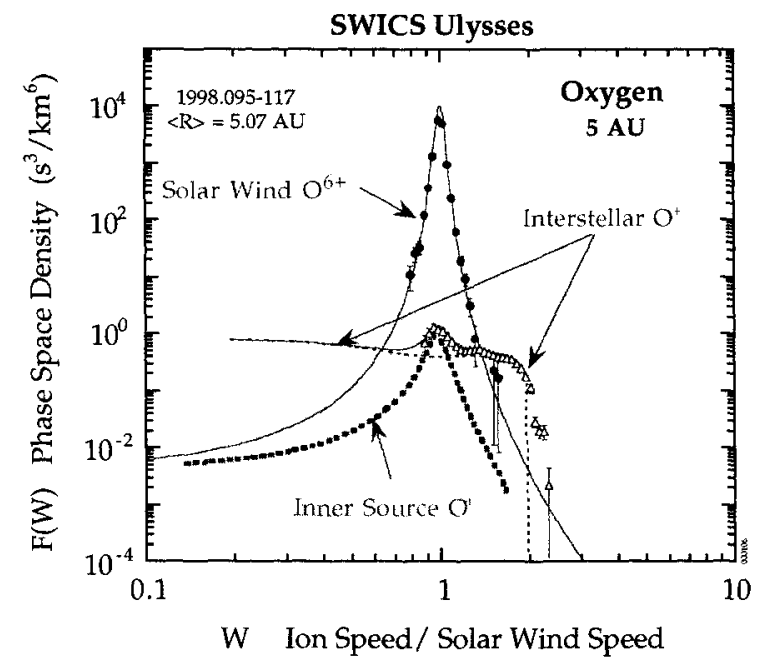

FIGURE 10. Comparison of the predicted speed distributions of inner source $\mathrm{O}^{+}$and interstellar $\mathrm{O}^{+}$at $5 \mathrm{AU}$ with the solar wind $\mathrm{O}^{6+}$ spectrum measured with SWICS on Ulysses at 5.07 AU from April 5 to April 27, 1998.

source and interstellar $\mathrm{O}^{+}$with that of solar wind $\mathrm{O}^{6+}$, measured by Ulysses at $\sim 5.1 \mathrm{AU}$. Now the density of the interstellar pickup $\mathrm{O}^{+}$exceeds that of solar wind $\mathrm{O}^{6+}$ for $W>\sim 1.4$ and $W<\sim 0.6$. For ions, such as $\mathrm{C}^{+}$, not present in the interstellar source, the inner source may still contribute even at $5 \mathrm{AU}$ if the injection efficiency is small for $W>\sim 0.1$.

\section{SUMMARY AND CONCLUSIONS}

One of our most important findings is that the speed distributions of $\mathrm{H}^{+}, \mathrm{He}^{+}$and $\mathrm{He}^{++}$in the in-ecliptic solar wind free of shocks and other disturbances have well developed high-speed tails indicating the presence of a population of highly suprathermal ions at all times. These tails are not formed in the corona since they are seen in the distributions of interstellar pickup $\mathrm{He}^{+}$. Furthermore, the density in the tails decreases no faster than $R^{-2}$ with distance $R$ from the Sun, as is most clearly seen in Figure 2 by comparing the $\mathrm{He}^{++}$tails measured at 1 and $5 \mathrm{AU}$. This implies that the quiet-time highspeed tails are continuously regenerated to overcome continuous cooling with increasing distance. Such cooling is clearly visible in the bulk solar wind which at $5 \mathrm{AU}$ has narrower peaks than at $1 \mathrm{AU}$ (see Figure 2).

One of the open questions then is how these ubiquitous ions, with speeds nearly ten times that of the solar wind, are continuously created in the low-latitude solar wind, and apparently not the high-latitude fast wind(15), at times when there are no shocks, waves or other solar wind disturbances observed locally. Because of their slow mobility, it is unlikely that these ions were accelerated by distant shocks and then transported to the shock free regions of the low-latitude heliosphere, although this possibility cannot be excluded. Fisk et al.(19) address this problem.

Downstream of shocks, suprathermal tails increase in strength by one to two orders of magnitude(15). The amount of the increase most likely depends on the strength of the shock. Our observation that the acceleration process produces spectra of similar shapes despite the large difference in the shock parameters once more suggests that the process responsible for the formation of tails is not due to the shocks themselves but rather to the turbulence in the downstream regions of the shocks as was also reported previously $(15,17,20)$. We suggest that the initial formation of the high-speed tails in the quiet solar wind and the subsequent strengthening of these tails in the turbulent solar wind downstream of shocks is the first step leading to acceleration of particles to energies above $\sim 0.1 \mathrm{MeV} / \mathrm{amu}$. In this sense, this initial step that energizes ions to many tens of $\mathrm{keV} / \mathrm{amu}$ could be considered the injection process.

We now come to the question of what material is injected, and then further accelerated to $\mathrm{MeV}$ energies. The fact that more $\mathrm{He}^{+}$than $\mathrm{He}^{++}$is observed above $W \approx 2$ both in the quiet in-ecliptic solar wind and in the turbulent regions of CIRs at $4-5 \mathrm{AU}(15)$ excludes the bulk solar wind, that is, around $W \approx 1$, as a significant source of CIR accelerated energetic particles. As shown in Figure 7, interstellar pickup ions, in particular $\mathrm{He}^{+}$, are efficiently injected and accelerated in CIRs. On the other hand, the injection efficiency of the thermal solar wind (ions within one thermal speed of the bulk speed) seems to be quite small $\left(<\sim 10^{-3}\right)$. Just how much of each of the three source populations (interstellar pickup ions, inner source pickup ions and suprathermal solar wind ions) is injected for further acceleration will depend on the relative fraction of these populations in the suprathermal range, where the injection efficiency becomes large, around $w>0.5$.

The mix of sources will also change with heliocentric distance (see Figures 8, 9 and 10) and with solar wind speed. Thus, the interstellar source should become dominant in the outer heliosphere at distances beyond 5 
to $10 \mathrm{AU}$. The inner source may be as important as the suprathermal solar wind in the inner heliosphere, from $\sim 0.5$ to $\sim 2$ to $4 \mathrm{AU}$. Also, when the solar wind speed is high, interstellar pickup ion distributions extend to higher speeds, and at twice the solar wind speed they may again have densities comparable to or higher than those of suprathermal solar wind ions at $W \approx 2$. It seems to be the case that in CIRs at $\sim 3-6 \mathrm{AU}$, the heated solar wind distributions for $\mathrm{H}, \mathrm{He}$ and $\mathrm{O}$ and the corresponding interstellar pickup ion distributions have roughly comparable densities near $W \approx 2$ and therefore relatively small changes in either the solar wind thermal speed or its bulk speed will affect the proportion of solar wind versus pickup ions accelerated in CIRs. Observations of the larger relative abundance of energetic $(\mathrm{MeV})$ $\mathrm{H}$ and He particles in CIRs (e.g. ref. 14) compared to that in the solar wind support our conclusion that pickup ions form a significant part of the seed population of energetic particles accelerated in these turbulent regions.

Finally, we come to the question of acceleration and of the source population of ACRs. It is well established that the source of the main components of ACRs are interstellar pickup ions(21). Inner source pickup ions, however, could well be the source for the rare ACRs(18) ( $\mathrm{C}, \mathrm{Mg}, \mathrm{Si}, \mathrm{S}$ and $\mathrm{Fe}$ ). While much of the acceleration to hundreds of $\mathrm{MeV}$ is likely to take place at the heliospheric termination shock, some acceleration of pickup ions inside the heliosphere is required in order to compensate for cooling with increasing heliocentric distance. The same mechanisms that produce suprathermal tails in the quiet low-latitude solar wind in the absence of shocks could also accelerate interstellar and inner source pickup ions in the outer heliosphere right up to the termination shock. Another possibility, suggested by Fisk(22), is acceleration at distances beyond $\sim 10 \mathrm{AU}$ at the interface between the low-speed solar wind in the ecliptic and the high-speed wind from the polar coronal holes at higher latitudes. Presumably, pickup ions in the turbulence associated with this interface would be accelerated as efficiently as in CIRs. Both process should be solar-cycle dependent. Highvelocity tails seem to be most pronounced in the slow solar wind, which dominates the heliosphere at solar maximum. The slow-fast wind interface, on the other hand, is most extensive at solar minimum, characterized by the permanent high-speed wind from the large coronal holes.

\section{ACKNOWLEDGMENTS}

We gratefully acknowledge the essential contributions of the many individuals (see Gloeckler et al.(1,2)) at the University of Maryland, the University of Bern, the Max-Planck-Institute fur Aeronomie and the Technical University of Braunschweig which assured the success of the SWICS experiments on ACE and Ulysses. Of particular benefit have been the many illuminating discussions with Johannes Geiss. We thank Christine Gloeckler for her help with data reduction. This work was supported in part by NASA/Caltech grant NAG56912 and NASA/JPL contract 955460.

\section{REFERENCES}

1. Gloeckler, G., et al., Space Sci. Revs. 86, 495-537 (1998).

2. Gloeckler, G., et al., Astron. Astrophys. Suppl. Ser. 92, 267-289 (1992).

3. Vasyliunas, V. M., and Siscoe, G. L., J. Geophys. Res. 81, 1,247-1,252 (1976).

4. Gloeckler, G., Space Sci. Revs. 78, 335-346 (1996).

5. Gloeckler, G., Fisk, L. A., and Geiss, J., Nature 386, 374-377 (1997).

6. Gloeckler, G., and Geiss, J., Space Sci. Revs. 86, $127-$ 159 (1998).

7. Gloeckler, G., Schwadron, N. A., Fisk, L. A., and Geiss, J., Geophys. Res. Lett. 22, 2665-2668 (1995).

8. Geiss, J., Gloeckler, G., Fisk, L. A., and von Steiger, R., J. Geophys. Res. 100, 23,373-23,377 (1995).

9. Geiss, J., Gloeckler, G., and von Steiger, R., Space Sci. Revs. 78, 43-52 (1996).

10. Gloeckler, G., Fisk, L. A., Geiss, J., Schwadron, N. A., and Zurbuchen, T. H., J. Geophys. Res. 105, 7459-7463 (2000).

11. Schwadron, N. A., Geiss, J., Fisk, L. A., Zurbuchen, T. H., and Gloeckler, G., J. Geophys. Res. 105, 74657472 (2000).

12. Gloeckler, G., et al., Nature (in press).

13. Keppler, E., Surveys in Geophysics 19, 211-278 (1998).

14. Mason, G. M., and Sanderson, T. R., Space Sci. Revs. 89, 77-90 (1999).

15. Gloeckler, G., Space Sci. Revs. 89, 91-104 (1999).

16. Balogh, A., et al., Space Sci. Revs. 72, 171-180 (1995).

17. Gloeckler, G., et al., J. Geophys. Res., 99, 17,63717,643 (1994).

18. Cummings, A. C., Stone, E. C., and Steenberg, C. D., "Composition of Anomalous Cosmic Rays and Other Ions from Voyager Observations", in Proceedings of the 26th International Cosmic Ray Conference 7, Salt Lake City, Utah, 1999, 531-534.

19. Fisk, L. A., Gloeckler, G., Zurbuchen, T. H., and Schwadron, N. A., "Statistical Acceleration of Suprathermal Particles in the Solar Wind", in these Proceedings of the ACE 2000 Symposium, 2000.

20. Schwadron, N. A., Fisk, L. A., and Gloeckler, G., Geophys. Res. Lett. 23, $2871-2874$ (1996).

21. Fisk, L. A., Kozlovsky, B., and Ramaty, R., Astrophys J. Lett. 190, L35-L38 (1974).

22. Fisk, L. A., Space Sci. Revs. 78, 129-136 (1996). 